\title{
8.5. CYCLIC VECTORS IN SPACES OF ANALYTIC FUNCTIONS*
}

Let $X$ be a Banach space of analytic functions in $\mathbb{D}$ satisfying the following two conditions: (i) for each $\zeta, \zeta \in \mathbb{D}$, the map $\mathrm{f} \rightarrow \mathrm{f}(\zeta)$ is a bounded linear functional on $X$; ( $i \mathrm{i}$ ) $z X \subset X$. It follows from (ii), by means of the closed graph theorem, that multiplication by $z$ is a bounded linear transformation (more briefly, an operator) on $X$. Finally, $f \in X$ is said to be a cyclic vector for the operator of mutiplication by $z$ if the finite linear com$b i n a t i o n s$ of the vectors $f, z f, z^{2} f, \ldots$ are dense in $X$ (when the constant function $f$ is in $\mathrm{X}$, one also says that $\mathrm{f}$ is weakly invertible in $\mathrm{X}$ ); this terminology was first used in [1].

Question 1. Does strong invertibility imply weak invertibility? (That is, if 1 , 1/f, $f$ are $\operatorname{all}$ in $X$, is $f$ cyclic?).

Consider the special case when $X$ is the Bergman space, i.e., the set of square-integrable analytic functions: $\|f\|^{2}=\int_{\mathbb{D}}|f|^{2}<\infty$.

Conjecture 1. If $f$ is in the Bergman space and if $|f(z)|>c(I-|z|)^{\text {a }}$ for some $c$, $\alpha>0$, then $f$ is cyclic.

If correct this would imply an affirmative answer to Question 1 when $X$ is the Bergman space. The conjecture is known to be correct under mild additional assumptions (see [2-4]). In particular it is correct when $\mathrm{f}$ is a singular inner function. In this case the condition in the hypothesis of the conjecture is equivalent to the condition that the singular measure associated with $f$ has modulus of continuity $O(\delta \log 1 / \delta)$ (see [1]).

Conjecture 2. A singular inner function is cyclic in the Bergman space if and only if its associated singular measure puts no mass on any Carleson set. (For the definition of Carleson set see [5], pp. 326-327.) $\dagger$

For more discussion of the cyclicity of inner functions see Sec. 6 of [6], pp. 54-58, where the possibility of an "inner-outer" factorization for inner functions is considered.

Question 2. Does there exist a Banach space of analytic functions, satisfying (i) and (ii), in which a function $f$ is cyclic if and only if it has no zeros in $\mathbb{D}$ ?

Nikolskii has shown [7] that no weighted sup-norm space of a certain type has this property. If such a space $X$ existed then the operator of multiplication by $z$ on $X$ would have the property that its set of cyclic vectors is nonempty and is a closed subset of space $X \backslash$ \{0\} (this follows since the limit of nonvanishing analytic functions is either nonvanishing or identically zero). No example of an operator with this property is known. (This may no longer be correct; Per Enflo. has announced an example of an operator on a Banach space with no invariant subspaces; i.e., every nonzero vector is cyclic. The construction is apparently exceedingly difficult.) Shapiro has shown that for any operator the set of cyclic vectors is always a $G_{\delta}$ set (see [8], Sec. 11, Proposition 40, p. 110). For a discussion of some of these questions from the point of view of weighted shift operators, see [8], Secs. 11, 12.

Question 3. Let $X$ be as before, and let $f, g \in X$ with $g$ cyclic. If $|f(z)| \geqslant|g(z)|$ in $\mathbb{D}$, is $\mathrm{f}$ cyclic?

This question has a trivial affirmative answer in spaces like the Bergman space, since bounded analytic functions multiply the space into itself. It is unknown for the Dirichlet space (i.e., the space of functions with $\left.\int_{\mathbb{D}}\left|f^{\prime}\right|^{2}<\infty\right)$; the special case $g$ = constant is estab-
lished in [9].

* ALLEN L. SHIELDS. Department of Mathematics, University of Michigan, Ann Arbor, Michigan 48109 .

tor else p. $242-\mathrm{Ed}$. 
1. Harold S. Shapiro, "Weakly invertible elements in certain function spaces and generators in $2^{1}$," Mich. Math. J., 11, 161-165 (1964).

2. Harold S. Shapiro, "Weighted polynomial approximation and boundary behavior of holomorphic functions," in: Contemporaty Problems in the Theory of Analytic Functions, Nauka, Moscow (1966), pp. 326-335.

3. G. Shapiro (H. S. Shapiro), "Some observations concerning the weighted polynomial approximation of holomorphic functions," Mat. Sb., 73, 320-330 (1967).

4. D. Aharonov, H. S. Shapiro, and A. L. Shields, "Weakly invertible elements in the space of square-summable holomorphic functions," J. London Math. Soc., No. 9, 183-192 (1974).

5. L. Carleson, "Sets of uniqueness for functions regular in the unit circle," Acta Math., $87,325-345(1952)$.

6. P. L. Duren, B. W. Romberg, and A. L. Shields, "Linear functionals on $\mathrm{H}^{\mathrm{P}}$ spaces with $0<\mathrm{p}<1, " \mathrm{~J}$. Reine Angew. Math., 238, 32-60 (1969).

7. N. K. Nikolskii, "The spectral synthesis and the problem of weighted approximation in spaces of analytic functions," Izv. Akad. Nauk Arm. SSR, Ser. Mat., 6, No. 5, 345-367 (1971).

8. Allen L. Shields, "Weighted shift operators and analytic function theory," in: Topics in Operator Theory, Math. Surveys No. 13, 49-128, Amer. Math. Soc., Providence (1974).

9. Allen L. Shields, "Cyclic vectors in some spaces of analytic functions," Proc. R. Ir. Acad. Sec. A, 74, 293-296 (1974). 